\title{
A TBX5 3'UTR variant increases the risk of congenital heart disease in the Han Chinese population
}

\author{
Feng Wang ${ }^{1,8}$, Dong Liu $^{2,8}$, Ran-Ran Zhang ${ }^{1}$, Li-Wei Yu ${ }^{1}$, Jian-Yuan Zhao ${ }^{3}$, Xue-Yan Yang ${ }^{3}$, \\ Song-Shan Jiang ${ }^{4}$, Duan Ma ${ }^{5}$, Bin Qiao ${ }^{6}$, Feng Zhang ${ }^{7}$, Li Jin ${ }^{3}$, Yong-Hao Gui ${ }^{1, *}$, Hong-Yan Wang ${ }^{7, *}$ \\ ${ }^{1}$ Children's Hospital, State Key Laboratory of Genetic Engineering at School of Life Sciences, Institute of Reproduction \& \\ Development, Fudan University, Shanghai, China; ${ }^{2}$ Co-innovation Center of Neuroregeneration, Key Laboratory of Neuror- \\ egeneration of Jiangsu and Ministry of Education, Nantong University, Nantong, China; ${ }^{3}$ The State Key Laboratory of Genetic \\ Engineering, Collaborative Innovation Center of Genetics and Development, School of Life Sciences, Fudan University, Shanghai, \\ China; ${ }^{4}$ The State Key laboratory for Biocontrol and MOE Key Laboratory of Gene Engineering, School of Life Sciences, \\ Sun Yat-Sen University, Guangzhou, China; ${ }^{5}$ Key Laboratory of Molecular Medicine, Ministry of Education, Department of \\ Biochemistry and Molecular Biology, Institute of Medical Sciences, Shanghai Medical College, Fudan University, Shanghai, \\ China; ${ }^{6}$ Institute of Cardiovascular Disease, General Hospital of Jinan Military Region, Jinan, China; ${ }^{7}$ The Obstetrics \& \\ Gynecology Hospital, Key Laboratory of Reproduction Regulation of NPFPC, Institute of Reproduction \& Development, Fudan \\ University, Shanghai, China
}

TBX5 is a vital transcription factor involved in cardiac development in a dosage-dependent manner. But little is known about the potential association of $T B X 53^{\prime}$ untranslated region (UTR) variations with congenital cardiac malformations. This study aimed to investigate the relationship between $T B X 53^{\prime}$ UTR variants and risk for congenital heart disease (CHD) susceptibility in two Han Chinese populations, and to reveal its molecular mechanism. The relationship between TBX5 $3^{\prime}$ UTR variants and CHD susceptibility was examined in 1177 CHD patients and 990 healthy controls in two independent case-control studies. Variant rs6489956 $\mathrm{C}>\mathrm{T}$ was found to be associated with increased $\mathrm{CHD}$ susceptibility in both cohorts. The combined CHD risk for the CT and TT genotype carriers was 1.83 times higher than that of CC genotype, while the risk for CT or TT genotype was 1.94 times and 2.31 times higher than that of CC carriers, respectively. Quantitative real-time PCR and western blot analysis showed that $\mathrm{T}$ allele carriers exhibited reduced $T B X 5 \mathrm{mRNA}$ and protein levels in CHDs tissues. Compared with $\mathrm{C}$ allele, $\mathrm{T}$ allele showed increased binding affinity to miR-9 and miR-30a in both luciferase assays and surface plasmon resonance analysis. Functional analysis confirmed that miR-9 and miR-30a downregulated $T B X 5$ expression at the transcriptional and translational levels, respectively. The assays in zebrafish model were in support of the interaction of miR-9/30a and TBX5 3'UTR (C and T allele). We concluded that TBX5 $3^{\prime}$ UTR variant rs6489956 increased susceptibility of $\mathrm{CHD}$ in the Han Chinese population because it changes the binding affinity of two target miRNAs that specifically mediate $T B X 5$ expression.

Keywords: congenital heart disease; TBX5; variant; 3'UTR; microRNAs

Cell Discovery (2017) 3, 17026; doi:10.1038/celldisc.2017.26; published online 25 July 2017

\section{Introduction}

Congenital heart diseases (CHDs) are the most prevalent type of human structural birth defects worldwide,

\footnotetext{
${ }^{8}$ These authors contributed equally to this work.

*Correspondence: Hong-Yan Wang

Tel: +862151630611; Fax: +862151630611

E-mail: wanghyan@fudan.edu.cn

or Yong-Hao Gui

E-mail: yhgui@shmu.edu.cn

Received 2 May 2017; accepted 29 June 2017
}

affecting between 19 and 75 per thousand live births [1]. The etiology of CHDs includes both genetic and environmental factors. Extensive model organism studies and linkage analysis of rare Mendelian CHD families have provided insights into the genetic basis of CHD, which results from alterations in transcription factors and genes involved in cardiac development [2-5]. However, mutations in these genes can only explain a small fraction of CHD cases. The pathogenesis of sporadic cases, the most common form of CHD, remains poorly understood. 
Table 1 Association between rs6489956 in the 3'UTR region of TBX5 and risk of congenital heart disease in two independent casecontrol studies

\begin{tabular}{|c|c|c|c|c|c|c|}
\hline \multirow[t]{2}{*}{ Group } & \multirow[t]{2}{*}{ GenotypelAllele } & \multirow[t]{2}{*}{ Control } & \multirow[t]{2}{*}{ Case } & \multicolumn{2}{|c|}{ Logistic regression } & \multirow[t]{2}{*}{$H W E p^{\mathrm{a}}$} \\
\hline & & & & OR $(95 \% C I)$ & $\mathrm{P}-$ value $^{\mathrm{b}}$ & \\
\hline \multirow[t]{4}{*}{ Shandong } & $\mathrm{C} / \mathrm{C}$ & $534(88.1 \%)$ & $703(77.7 \%)$ & 1.00 & $1.65 \times 10^{-7}$ & 0.085 \\
\hline & $\mathrm{C} / \mathrm{T}-\mathrm{T} / \mathrm{T}$ & $72(11.9 \%)$ & $202(22.3 \%)$ & $2.33(1.70-3.19)$ & & \\
\hline & $\mathrm{C}$ & $1135(93.6 \%)$ & $1595(88.1 \%)$ & 1.00 & $2.86 \times 10^{-7}$ & \\
\hline & $\mathrm{T}$ & $77(6.4 \%)$ & $215(11.9 \%)$ & $2.16(1.61-2.91)$ & & \\
\hline \multirow[t]{4}{*}{ Shanghai } & $\mathrm{C} / \mathrm{C}$ & $318(82.8 \%)$ & $197(72.4 \%)$ & 1.00 & 0.0013 & 0.54 \\
\hline & $\mathrm{C} / \mathrm{T}-\mathrm{T} / \mathrm{T}$ & $66(17.2 \%)$ & $75(27.6 \%)$ & $1.75(1.24-2.46)$ & & \\
\hline & $\mathrm{C}$ & $698(90.9 \%)$ & $462(84.9 \%)$ & 1.00 & 0.001 & \\
\hline & $\mathrm{T}$ & $70(9.1 \%)$ & $82(15.1 \%)$ & $1.77(1.26-2.49)$ & & \\
\hline \multirow[t]{4}{*}{ Combined } & $\mathrm{C} / \mathrm{C}$ & $852(86.1 \%)$ & $900(76.5 \%)$ & 1.00 & $3.62 \times 10^{-8}$ & 0.1 \\
\hline & $\mathrm{C} / \mathrm{T}-\mathrm{T} / \mathrm{T}$ & $138(13.9 \%)$ & $277(23.5 \%)$ & $1.83(1.48-2.28)$ & & \\
\hline & $\mathrm{C}$ & $1833(92.6 \%)$ & $2057(87.4 \%)$ & 1.00 & $2.01 \times 10^{-8}$ & \\
\hline & $\mathrm{T}$ & $147(7.8 \%)$ & $297(12.6 \%)$ & $1.86(1.50-2.30)$ & & \\
\hline
\end{tabular}

Abbreviations: CI, confidence interval; HWE, Hardy-Weinberg equilibrium; OR, odds ratio.

${ }^{a} P$-value for HWE test in control subjects.

${ }^{\mathrm{b}}$ Adjusted for age and gender.

The bold entries indicate significant $P$ values.

Genome-wide association studies (GWASs) suggest that a large number of non-coding variants contribute to the risk of various diseases, including cardiovascular disease [610]. Our previous study demonstrated that non-coding variants in genes coding for enzymes that biotransform homocysteine significantly increase CHD risk by regulating gene expression levels [11-13]. During embryonic cardiogenesis, many transcription factors including TBX5 [14], GATA4 [15] and NKX2-5 [16] are strictly regulated in a dosage-dependent manner. Mutations in the coding region of $T B X 5$ invariably result in Holt-Oram syndrome (HOS), an inherited disease characterized by upper limb and cardiac deformities $[2,17]$. Thus, we hypothesized that the functional regulatory variations in $T B X 5$ might be associated with increased CHD risk through altered gene expression or dosage effects.

TBX5 is a transcription factor with well-defined roles in heart and forelimb development. Multiple studies in animal models have confirmed that cardiac development is sensitive to Tbx5 dosage [14, 18, 19]. Most notably, CHD might result from the haploinsufficiency of Tbx5.

Recently, several non-coding variants of TBX5 in the promoter [20], intron [21] and enhancer regions [22] were reported to be associated with CHDs. Although the $3^{\prime}$ untranslated region $\left(3^{\prime} \mathrm{UTR}\right)$ is critically important for microRNAs (miRNAs) binding to regulate gene expression, the potential contribution of mutations in the TBX5 $3^{\prime} \mathrm{UTR}$ to CHDs remains unknown. Our previous study demonstrated that miR-10a and miR-10b significantly repressed $T B X 5$ protein levels by targeting its $3^{\prime}$ UTR [23]. In present study, we detected a common variant, rs6489956, in the $T B X 53^{\prime}$ UTR that significantly increases CHD risk in a Han Chinese cohort composed of 1177 CHD patients and 990 healthy controls. We further investigated the molecular mechanism underlying that TBX5 $3^{\prime} \mathrm{UTR}$ variants influence risk for CHD susceptibility.

\section{Results}

TBX5 3'UTR variant rs6489956 significantly increased CHD susceptibility in Han Chinese

In our study, a total of ten variants in TBX5 $3^{\prime} \mathrm{UTR}$ were identified. Among them, four variants had minor allele frequency (MAF) $>0.05$ and were chosen for genotyping in 288 cases and controls of the Shandong group. Among them, the prevalence of variant rs6489956 (c. ${ }^{*} 1101 \mathrm{C}>\mathrm{T}$ ) was significantly different between the CHDs and the control subjects. This variant was then selected for further validation in the other two independent case-control cohorts (Supplementary Table S3).

As the homozygous TT genotype was very rare, we applied the dominant model of inheritance to enhance the statistical power of the study, which combined homozygous TT alleles with heterozygous CT alleles to compare with the wild-type CC alleles in the association studies. Logistic regression analyses demonstrated heterozygote CT and homozygote TT subjects had a 
Table 2 Stratified analysis of rs6489956 by CHDs classification

\begin{tabular}{|c|c|c|c|}
\hline & No. & P-value & $\begin{array}{c}\text { Association }(O R(95 \% C I))^{\mathrm{a}} \\
C T / T T \text { vs } C C\end{array}$ \\
\hline \multicolumn{4}{|l|}{ CHDs classification $I^{\mathrm{b}}$} \\
\hline Conotruncal defects & 156 & $4.63 \times 10^{-5}$ & $2.35(1.56-3.54)$ \\
\hline Septal defects & 830 & $1.71 \times 10^{-6}$ & 1.85 (1.44-2.39) \\
\hline LVOTO & 17 & 0.171 & $2.27(0.70-7.29)$ \\
\hline RVOTO & 24 & 0.114 & $2.14(0.83-5.52)$ \\
\hline APVR & 16 & 0.94 & $0.94(0.21-4.25)$ \\
\hline Complex CHDs & 14 & 0.025 & 3.58 (1.17-10.93) \\
\hline Other cardiac abnormalities & 120 & 0.006 & $2.22(1.26-3.93)$ \\
\hline \multicolumn{4}{|l|}{ CHDs classification II } \\
\hline Isolated CHDs & 1016 & $6.67 \times 10^{-7}$ & $1.85(1.45-2.36)$ \\
\hline Non-isolated CHDs & 161 & $9.83 \times 10^{-7}$ & $2.68(1.81-3.98)$ \\
\hline \multicolumn{4}{|l|}{ Detailed CHDs phenotypes } \\
\hline ASD & 120 & $3.67 \times 10^{-3}$ & $2.00(1.25-3.18)$ \\
\hline VSD & 691 & $2.69 \times 10^{-5}$ & $1.76(1.35-2.30)$ \\
\hline TOF & 98 & 0.031 & $1.78(1.06-3.02)$ \\
\hline
\end{tabular}

significantly increased risk of CHD compared with those with the wild-type CC allele in a study of 905 CHDs and 606 controls in the Shandong group (odds ratio $(\mathrm{OR})=2.33, \quad 95 \% \quad$ confidence interval $(\mathrm{CI})=1.70-3.19, P=1.65 \times 10^{-7}$ ), as well as $272 \mathrm{CHDs}$ and 384 controls in the Shanghai cohort $(\mathrm{OR}=1.75,95 \%$ $\mathrm{CI}=1.24-2.46, P=0.0013$ ). The combination of these two independent studies showed a 1.83 -fold increased CHD risk when the dominant model is used for $T B X 5$ rs6489956 $\left(P=3.62 \times 10^{-8}\right)$ in a total number of 1177 CHDs patients and 990 controls (Table 1), with an adjusted OR of $1.94(95 \% \quad \mathrm{CI}=1.52-2.47$, $\left.P=8.21 \times 10^{-8}\right) \quad$ and $2.31 \quad(95 \% \quad \mathrm{CI}=1.01-5.30$, $P=0.033$ ) for the CT and TT genotypes, respectively. Therefore, The variant rs6489956 was significantly associated with an increased risk of CHD in two cohorts separately and together. Similarly, the allele analysis showed that carriers of the susceptible $\mathrm{T}$ allele exhibited a higher risk of $\mathrm{CHD}$ than carriers of the $\mathrm{C}$ allele in both cohorts, resulting in a 1.86-fold combined higher susceptibility of CHD $\quad(95 \% \mathrm{CI}=1.50-2.30$, $\left.P=2.01 \times 10^{-8}\right)$. All genotype frequencies were in accordance with the Hardy-Weinberg expectation among the control subjects $(P>0.05)$.

\section{TBX5 c. *1101C $>T$ was highly related to septation defects}

A stratified analysis was performed according to standard CHDs classifications as previously described $[1,24]$. It was observed that TBX5 c. ${ }^{*} 1101 \mathrm{C}>\mathrm{T}$ (rs6489956) is related to multiple types of CHDs (Table 2). Notably, the highest statistical significance was observed in 830 patients with septal defects (heterozygous $\mathrm{CT}$ with adjusted $\mathrm{OR}=1.82, \quad 95 \%$ $\mathrm{CI}=1.41-2.37$; homozygous $\mathrm{TT}$ with adjusted $\mathrm{OR}=$ $2.28,95 \% \mathrm{CI}=0.95-5.49 ; P=9.70 \times 10^{-6}$ ). In isolated phenotypes, similar statistical significance was noted in atrial septal defect (ASD) cases $\left(P=3.67 \times 10^{-3}\right)$ and ventricular septal defect (VSD) cases $\left(P=2.69 \times 10^{-5}\right)$.

\section{The TBX5 c. ${ }^{*} 1101 C>T$ affected TBX5 mRNA transcription and translation}

Since the $T B X 5$ c.* ${ }^{*} 1101 C>\mathrm{T}$ (rs648995) variant is located in the TBX5 $3^{\prime} \mathrm{UTR}$, we assumed that this 
a

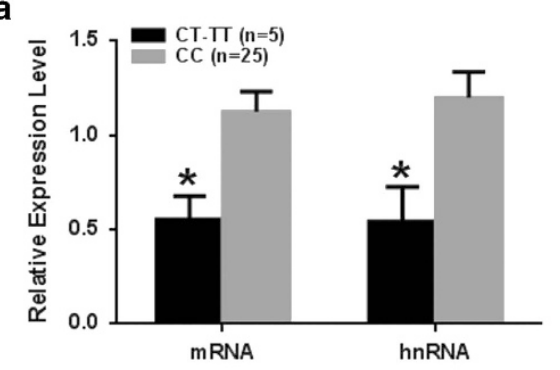

b

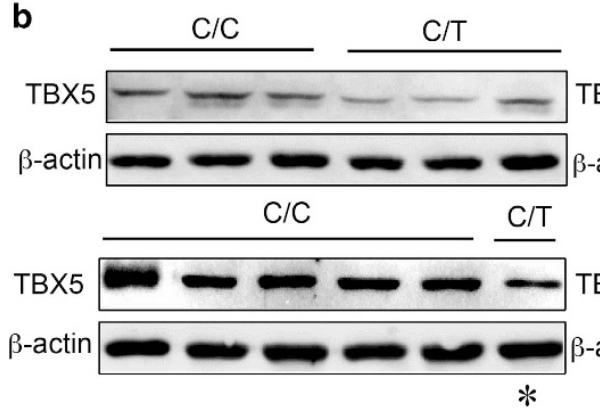

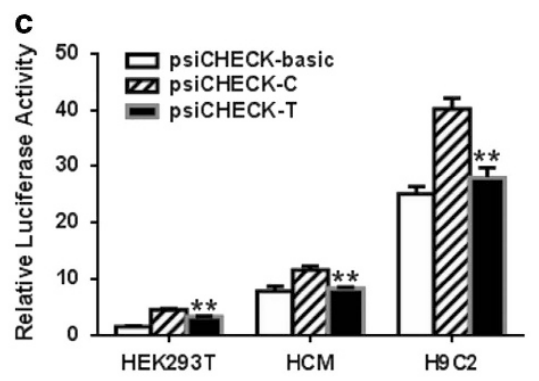

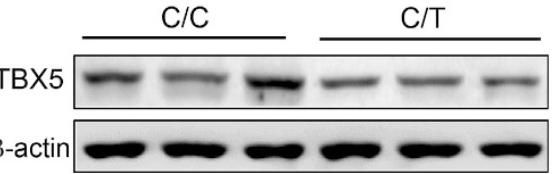

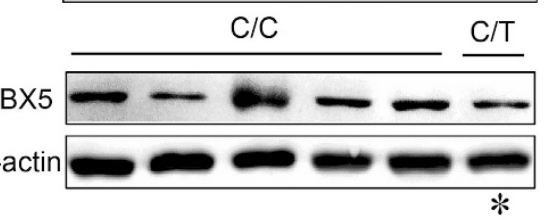

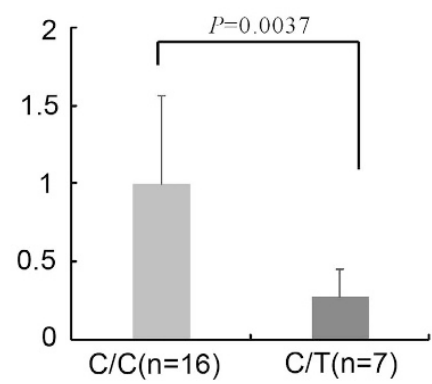

Figure 1 The T allele of rs6489956 reduced TBX5 mRNA and protein expression levels in vivo and in vitro. (a) Quantitative realtime PCR analysis of TBX5 mRNA levels in 30 cardiac tissue samples from individuals carrying different rs6489956 C $>$ T genotypes. The values for each genotype group were: $C C=1.12 \pm 0.53$ (mRNA), $1.20 \pm 0.67$ (heterogenous nuclear RNA, hnRNA), CT/TT $=0.55 \pm 0.27$ (mRNA), $0.54 \pm 0.41$ (hnRNA). All values were normalized to the levels of $\beta$-actin and represent the mean \pm s.d. of three independent experiments. ${ }^{*} P<0.05$. (b) Western Blot analysis in 23 cardiac tissue samples of $\mathrm{CHDs}$ indicated that TBX5 protein level in the CT genotype was only $27.1 \%$ that of the CC genotype $(P<0.01)$. Considering the rare samples of the CT genotype, one of the seven tissues was used twice to compare with the CC genotype and denoted as *. (c) Luciferase expression was significantly decreased in the minor $\mathrm{T}$ allelic reporter compared with the major $\mathrm{C}$ reporter in different cells. The values in HEK 293T cells were: psiCHECK-basic $=1.61 \pm 1.20$, psiCHECK-C $=4.60 \pm 0.39$, psiCHECK$\mathrm{T}=3.38 \pm 0.22$; the values in $\mathrm{HCM}$ cells were: psiCHECK-basic $=7.85 \pm 1.55$, psiCHECK-C $=11.64 \pm 2.03$, psiCHECK$\mathrm{T}=8.31 \pm 0.58$; the values in $\mathrm{H} 9 \mathrm{C} 2$ cells were: psiCHECK-basic $=25.12 \pm 3.09$, psiCHECK-C $=40.18 \pm 5.78, \mathrm{psiCHECK}-\mathrm{T}$ $=28.03 \pm 5.35$. Each value represented the mean \pm s.d. of three independent experiments. ${ }^{* *} P<0.01$.

variant might be involved in either $T B X 5$ transcription or translation. Thus, we examined TBX5 mRNA expression using quantitative RT-PCR on the cardiac tissue samples. Of the 30 cardiac samples tested, 25 had the rs6489956 CC genotype, four had the CT genotype, and only one had the TT genotype. The relative TBX5 mRNA expression level in the CT/TT genotypes was only $49.3 \%$ of that of the CC genotype $(P<0.05)$ (Figure 1a). Consistent with the reduction in mRNA expression, TBX5 protein level in the CT genotype was reduced by $72.9 \%$ compared with the $\mathrm{CC}$ genotype
$(0.27 \pm 0.19$ vs $1.0 \pm 0.57, P=0.0037)$ (Figure $1 b)$. Although this study measured TBX5 mRNA and protein levels in heart tissue samples from subjects who have already been born, which might be different from embryonic $T B X 5$ levels, our results demonstrated that $T B X 5$ transcription and translation levels were correlated with the variant rs6489956 genotypes.

Altered TBX5 mRNA expression associated with the variant rs6489956 was further confirmed by in vitro luciferase assays in different cell lines. Consistent with the tissue sample results, the plasmid containing the mutant-T allele 
a

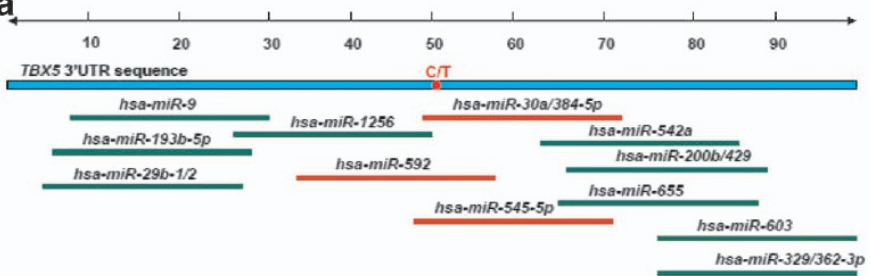

b

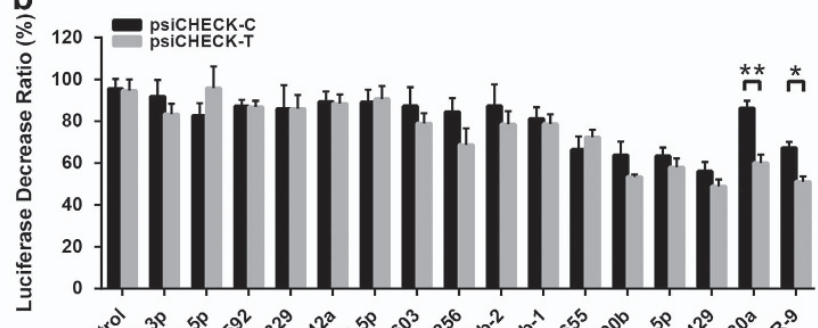

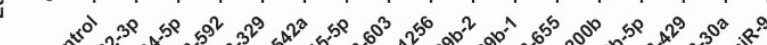
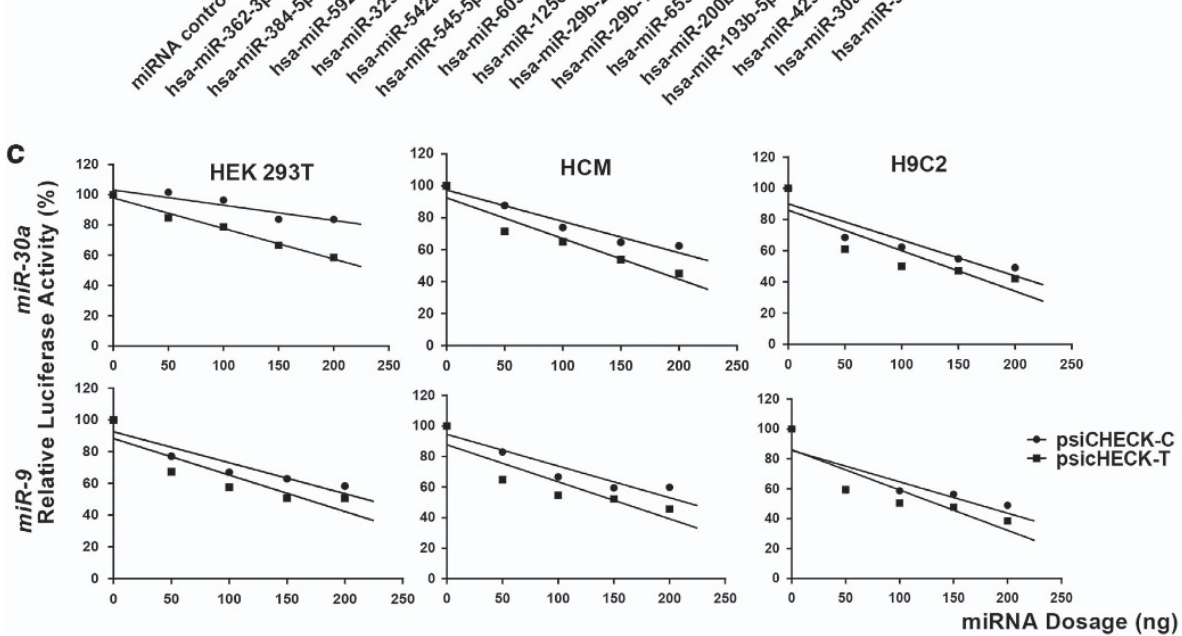

d

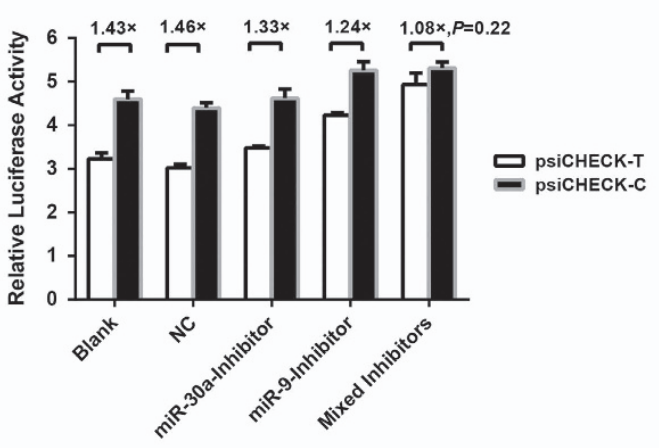

e
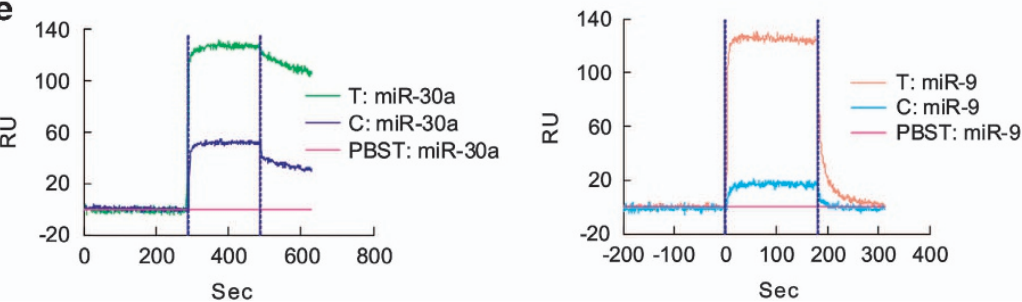
showed a $26 \%, 28 \%$ and $31 \%$ reduction in luciferase activity when compared with the wild-type C allele in HEK 293T, $\mathrm{HCM}$ and $\mathrm{H} 9 \mathrm{C} 2$ cells, respectively $(P<0.01)$ (Figure $1 \mathrm{c})$. Therefore, both in vivo and in vitro experimental evidence clearly supports the hypothesis that the $T B X 5 \mathrm{c} .{ }^{*} 1101 \mathrm{C}>\mathrm{T}$ variant affected the transcription of $T B X 5$.

miR-9 and miR-30a inhibited TBX5 expression by interacting with the TBX5 $c{ }^{*} 1101 C>T$ variant

In the flanking region of the TBX5 $3^{\prime} \mathrm{UTR}$ variant rs6489956, there are 16 software-predicted miRNAs including five upstream miRNAs, seven downstream miRNAs and four on-site miRNAs (Figure 2a). It was found that only 2 out of these 16 miRNAs, namely, miR-9 and miR-30a, can cause a significant disparity in the expression of the two allelic reporters in HEK $293 \mathrm{~T}$ cells co-transfected with the psiCHECK-C/T plasmid (Figure 2b). The endogenous expression of miR-9 and miR-30a was detected in all of the 30 human cardiac samples (Figure 3a). We also observed that miR-30a binds to $T B X 5$ mRNA carrying the $\mathrm{T}$ allele with a higher affinity compared with that of the $\mathrm{C}$ allele $\left(5.10 \times 10^{-7} \mathrm{moll}^{-1}[\mathrm{M}]\right.$ vs $\left.1.23 \times 10^{-6} \mathrm{M}\right)$ (Figure 2e). For the upstream miR-9, although the variant rs6489956 appears not to directly affect miR-9 binding (Supplementary Figure S1), it might impair the miRNAs complex that interacts with the TBX5 motif containing variant rs6489956, as previously described [25].

The specific inhibition of miR-9 and miR-30a for TBX5 caused by the $3^{\prime}$ UTR variant c. ${ }^{*} 1101 \mathrm{C}>\mathrm{T}$ was demonstrated by a psiCHECK luciferase reporter assay as well as the rescue experiments from miRNA inhibitors. The luciferase gene expression of psiCHECK-C and psiCHECK-T were both inhibited in a linear manner with increased miRNAs concentration, and the miRNAs displayed a more pronounced inhibition effect on psiCHECK-T (Figure 2c). On the other hand, the allelic expression disparity was diminished by the use of each specific inhibitor for miR-9 or miR-30a individually or in tandem (Figure 2d).
miR-9 and miR-30a inhibited TBX5 expression at the transcriptional and translational levels independently

It was determined that both miR-9 and miR-30a were present at detectable levels in cardiac tissues (Figure 3a), and the expression of the two miRNAs was 3.7 to 4.9 times higher in cardiac tissues from CHD cases compared with that of cardiac tissues from the non-CHD controls (Figure 3b). The cardiac tissue samples of CHD cases were collected from children (average 4.71 years old), and the non-CHD controls were collected from adults (average 32.7 years old). Despite a potential miRNAs expression disparity between adults and children, such a large difference in miR-9 and miR-30a expression might still represent the potential contributions of the two miRNAs in CHD development, which would require more robust studies to confirm in the future.

Because miR-9 and miR-30a had similar inhibition patterns on the luciferase expression vector with the TBX5 3'UTR (Supplementary Figure S2), we measured whether $T B X 5$ transcription or translation in cultured HEK 293 T cells could be regulated by miR-9 and miR-30a. MiR-9 reduced the transcription of TBX5 mRNA by $57 \%$ (Figure 3c, left), and the miR-9 inhibitor up-regulated TBX5 mRNA expression 1.88fold (Figure 3d, left). However, neither miR-30a nor its inhibitor had any effect on TBX5 mRNA expression (Figure $3 \mathrm{c}$ and d, left). Both miR-9 and miR-30a could decrease TBX5 translation (Figure 3c, right). Additionally, miR-9 or miR-30a inhibitors could increase TBX5 protein expression separately or together (Figure 3d, right). These results suggested that miR-9 primarily regulates endogenous transcription of $T B X 5$, and that miR-30a regulates translation of the $T B X 5$ mRNA. Therefore, the mechanisms by which miR-9 and miR-30a regulate $T B X 5$ were confirmed both in vitro and in vivo.

\section{TBX5 3'UTR variant rs6489956 interacted with miR-30a or miR-9 in zebrafish}

In order to test the interaction effect of TBX5 $3^{\prime}$ UTR variant and miR-30a or miR-9 in vivo, we explored their function on heart development using

Figure 2 Screen for miRNAs interacting with TBX5 c. ${ }^{*} 1101 \mathrm{C}>$ T variant. (a) 16 Software-predicted miRNAs adjacent to the TBX5 c. *1101C > T variant; (b) the luciferase assay showed that only two miRNAs, miR-30a and miR-9, had statistically significant differences between the two allelic reporters. ${ }^{*} P<0.05$, ${ }^{* *} P<0.01$. (c) Luciferase expression of psiCHECK-C/T was inhibited by miR-30a or miR-9 in a dose-dependent manner, and the inhibition effect was more pronounced with the $T$ allele. (d) The expression discrepancy between psiCHECK2-C and T was diminished by miRNA inhibitors, either separately or together; NC represents as negative control. (e) SPR analysis results showed the binding affinity of miR-30a or miR-9 to the TBX5 $3^{\prime}$ UTR T allele mRNA was higher than that of the C allele at the same concentration of $20 \mu \mathrm{M}$. RU: resonance units; Sec: second. 

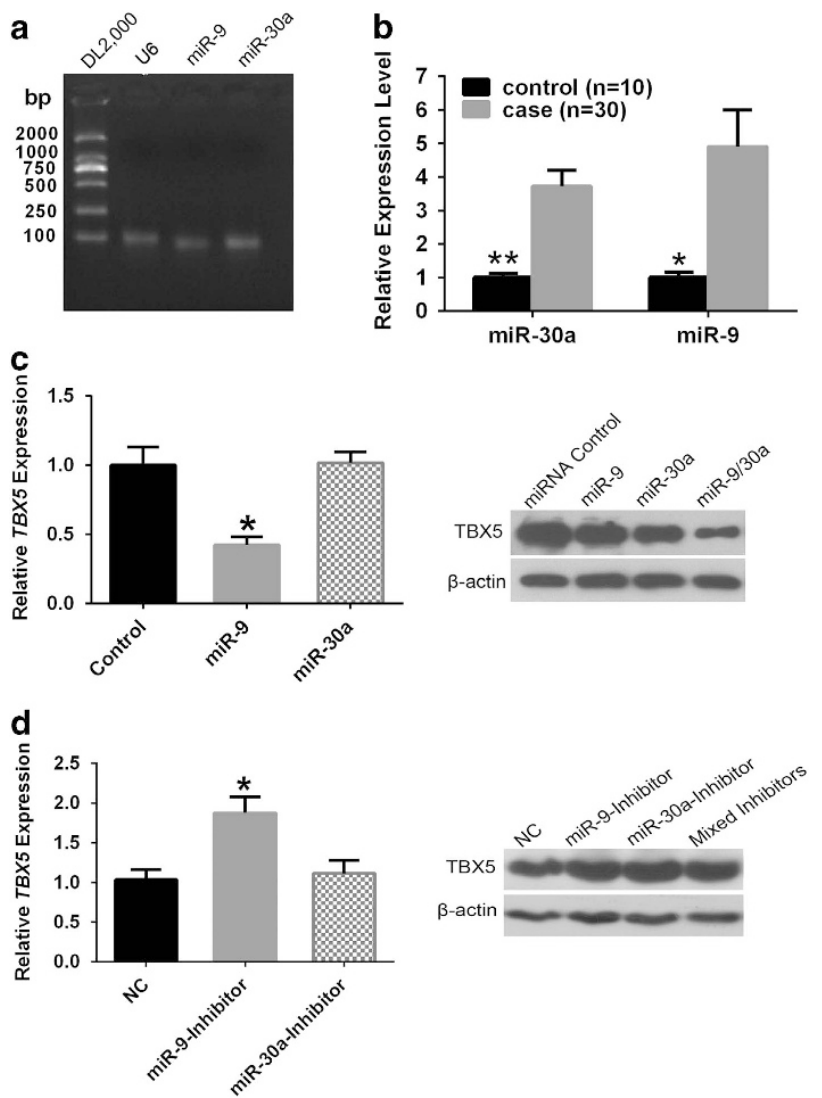

Figure 3 The functional validation of the candidate miRNAs. (a) RT-PCR showed that miR-9 and miR-30a were expressed in heart tissues. These tissues originated from the outlet samples of TOF patients during cardiac operation. (b) miR-30a and miR-9 expression levels in $30 \mathrm{CHDs}$ heart tissue samples were 3.73 and 4.91 times higher than the controls, respectively. The actual values compared to the internal reference gene levels for each miRNA were as follows: $\operatorname{miR}-30 a=0.096 \pm 0.071$, miR-9 $=0.0034 \pm 0.0005$; (c) TBX5 mRNA expression in HEK 293T cells was downregulated by transfection with miR-9 rather than miR-30a (left), while the presence of both miRNAs inhibited TBX 5 protein concentration according to the western blot analysis results (right). (d) TBX5 mRNA concentration increased in the cells transfected with miR- 9 inhibitor, but did not change following transfection of miR-30a inhibitor (left). In contrast, TBX5 protein concentration was up-regulated with transfection of the two miRNAs inhibitors. NC represents as negative control.

zebrafish model system. We set up 11 groups for microinjection and did imaging analysis at $48 \mathrm{~h}$ post fertilization (hpf): wild-type (WT) control, standard MO control (5 ng), tbx5 MO (5 ng), tbx5 MO (2.5 ng), tbx5 MO (0.5 ng), MO (5 ng)+C allele mRNA $(100 \mathrm{pg}), \mathrm{MO}(5 \mathrm{ng})+\mathrm{T}$ allele mRNA (100 pg), MO $(5 \mathrm{ng})+\mathrm{C}$ allele mRNA $(100 \mathrm{pg})+\mathrm{hsa}-\mathrm{mi} 9(100 \mathrm{pg}), \mathrm{MO}$ $(5 \mathrm{ng})+\mathrm{T}$ allele mRNA (100 pg)+hsa-miR-9 (100 pg), MO (5 ng)+C allele mRNA (100 pg)+hsa-miR-30a
$(100 \mathrm{pg})$ and $\mathrm{MO}(5 \mathrm{ng})+\mathrm{T}$ allele mRNA (100 pg)+hsamiR-30a (100 pg). As previously reported [26-29], tbx5 loss-of-function in zebrafish caused heart developmental defects, including different degrees of heart looping arrested, heart edematous, string-like heart, and so on, which were confirmed in our results (Figure $\left.4 \mathrm{a}-\mathrm{d}^{\prime}\right)$. The TBX5 3'UTR variant injection displayed partial rescue of loss-of-function effects in live embryos (Figure 4e). While co-injection of TBX5 3'UTR variant with miR-30a or miR-9 into tbx5 loss-of-function embryos significantly reduced the rescue effects, especially in $\mathrm{T}$ allele and miR-30a or miR-9 coinjected embryos (Figure 4e). The fluorescence sensor assay in zebrafish embryos demonstrated that the miR-9/30a precursor repressed the expression of egfp-tbx5-3'UTR-C and more apparently the expression of egfptbx5-3'-UTR-T in vivo (Figure 4f). The assays in zebrafish model were in support of the interaction of miR-9/30a and TBX5 3'UTR (C and T allele).

\section{Discussion}

It is well known that the mutations in $T B X 5$ gene coding regions have a role in syndromic CHD such as Holt-Oram syndrome [2, 30-36]. However, the role of $T B X 5$ in sporadic isolated cases of CHD has not been extensively explored. Experimental evidence based on animal models has confirmed that $T b x 5$ has vital roles in cardiac development, and that cardiac development is highly sensitive to $T b x 5$ dosage [14, 18, 37, 38]. Therefore, we believed that functional regulatory variants of $T B X 5$ might contribute to the etiology of sporadic isolated CHDs through a dosage effect. In this study, we investigated all common SNPs in the $T B X 5$ $3^{\prime}$ UTR region and focused on the potential functional variant that we thought might modulate TBX5 expression by its interaction with miRNAs. The functional variant $T B X 5$ c. ${ }^{*} 1101 \mathrm{C}>\mathrm{T}$ (rs6489956) was found to significantly increase CHD risk in Han Chinese by interacting with miR-30a and miR-9 (OR $=1.83 .95 \% \mathrm{CI}=1.48-2.28, P=3.62 \times 10^{-8}$ in the dominant model). Although we first postulated that a functional variant in the TBX5 3'UTR may increase the risk of CHD, a recent study indirectly supported our findings by reporting that a $T B X 5$ enhancer variant causes isolated congenital heart disease [22]. Because our study was only conducted among the Han Chinese subjects, such relationships between variants in the TBX5 $3^{\prime} \mathrm{UTR}$ and the risk of CHD need further exploration in other ethnic groups. However, our study elucidated that the sporadic CHDs could be attributed to the combined contribution of the common 

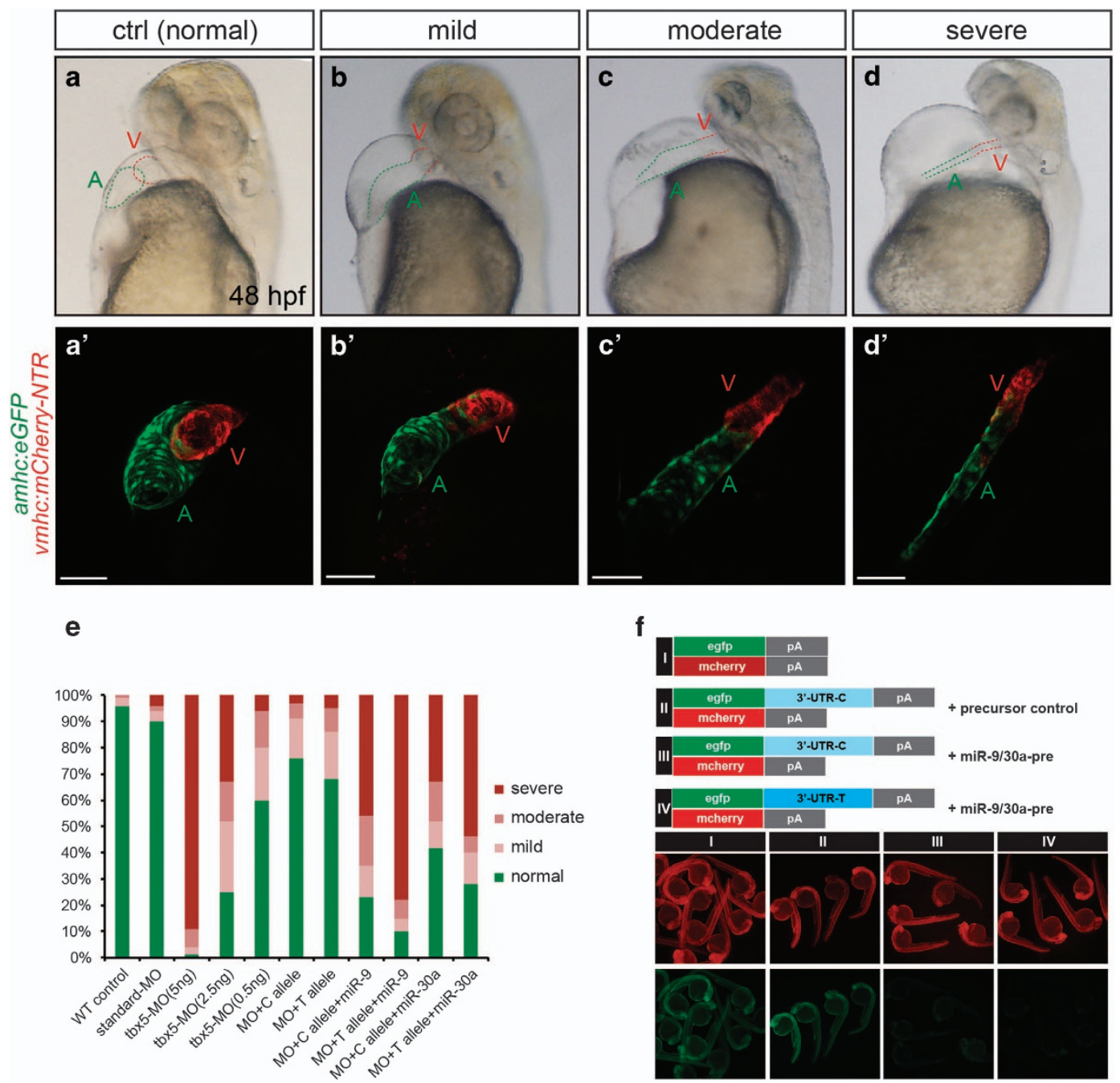

Figure 4 TBX5 3'UTR variant rs6489956 affected heart development in zebrafish embryo. (a-d') Examples of heart defects with variable severity in zebrafish: (a and $\mathbf{a}^{\prime}$ ) normal heart; ( $\mathbf{b}$ and $\mathbf{b}^{\prime}$ ) mild defect: heart shows mild looping arrested, pericardium effusion, and dropsy of ventral position; (c and $\left.\mathbf{c}^{\prime}\right)$ moderate defect: heart shows moderate looping arrested, pericardium effusion and dropsy of ventral position; ( $\mathbf{d}$ and $\mathbf{d}^{\prime}$ ) severe defect: malformed embryo with small, string-like heart, severe pericardium effusion, and dropsy of ventral position. Green $A$ indicates ventricle; red V indicates atrium. (e) Distribution of four categories of heart morphologies in each experimental group at $48 \mathrm{hpf}$ : WT control, MO standard control, $\mathrm{MO}(5 \mathrm{ng}), \mathrm{MO}(2.5 \mathrm{ng}), \mathrm{MO}$ (0.5 ng), $\mathrm{MO}+\mathrm{C}$ allele mRNA, MO+T allele mRNA, MO+C allele mRNA+miR-9, MO+T allele mRNA+miR-9, MO+C allele mRNA+miR-30a, $\mathrm{MO}+\mathrm{T}$ allele mRNA+miR-30a. (f) EGFP sensors were coinjected with mCherry control as indicated. miR-9/30a precursor injection reduced EGFP levels in EGFP-3'UTR sensor (third column), while mCherry levels were unchanged. In the EGFP-3' UTR-T sensor (fourth column), more significant reduction in EGFP was noted.

regulatory variations in the $T B X 5$ gene, as well as the epigenetic factors such as miR-30a or miR-9.

The possible mechanism of underlying the association of $T B X 5$ non-coding variants with increased CHD risk may be explained by the sensitive nature of the dosage effect of TBX5 on heart development. While severe loss-of-function mutations in $T B X 5$ coding regions led to both heart and forelimb phenotypes, non-coding variants were associated with compartmentalized phenotypes, which might reflect the differing sensitivities of the impacted tissues to the altered TBX5 expression level. This contention is supported by the fact that the $T B X 5$ intronic variant rs7312625 was reported to be associated with altered $\mathrm{PR}$ intervals (intervals between $\mathrm{P}$ wave and $\mathrm{R}$ wave in electrocardiogram). In African Americans, and intronic variant rs3825214 was shown to reduce lone atrial fibrillation risk in Han Chinese [39, 40]. The tissue- 
specific regulation of different non-coding regions could be one possible explanation.

In both embryonic and adult human heart tissues, $T B X 5$ expression can be observed throughout the epicardium and myocardium, including the free walls and septa of all four cardiac chambers, while its expression in the endocardium is limited to the left ventricle [41]. However, in mouse [42] and chicken [43], Tbx5 mRNA expression is restricted to the atria and left ventricle. Consistent with these expression patterns, HOS patients with $T B X 5$ mutations may have a number of significant heart defects, such as septal defects and certain complex malformations (tetralogy of Fallot (TOF), APVR and hypoplastic left heart) $[42,44]$. In our study of sporadic CHD cases, we observed that rs6489956 is correlated with a series of cardiac abnormalities; septal defects were statistically most common $\left(n=830, P=9.70 \times 10^{-6}\right)$. Additionally, we speculated that the failure to detect any correlation between variant rs6489956 and APVR and outflow tract malformation susceptibility might be due to the limited and insufficient sample size.

Both in vivo and in vitro assays on the function of variant rs6489956 indicated that the $\mathrm{T}$ allele led to reduction in TBX5 gene expression. We further identified two miRNAs, miR-9 and miR-30a, that interacted with this variant. This is not surprising, because miRNAs are predicted to function in the posttranscriptional regulation of nearly one third of all human genes [45]. It was confirmed that miR-30a did not affect the concentration of $T B X 5$ mRNA, but reduced its protein concentration, while miR-9 downregulated both transcription and translation of $T B X 5$. It has been extensively reported that miRNAs help to regulate cardiac growth and remodeling, and contribute to specific myocyte properties. miRNAs are implicated in numerous cardiovascular diseases, such as heart hypertrophy, heart failure, atherosclerosis, acute myocardial infarction, VSD, TOF and arrhythmias [46-52]. Thus, when the expression of the developmentally important transcription factor, $T B X 5$ was disturbed during a sensitive time window of development, it increases the likelihood of inducing CHD. As a new model trigger, the interactions between miRNAs and $T B X 5$ regulatory variant increase the CHD risk through the downregulated $T B X 5$, either at the posttranscriptional or translational level.

Because miRNAs are vital factors that mainly target 3'UTR sequences of mRNA, we suspected that miRNAs might have important roles in CHDs. Both bioinformatic predictions and functional analyses showed that both miR-30a and miR-9 have a higher affinity to the minor $\mathrm{T}$ allele. We further confirmed these results using luciferase assays, quantitative RT-PCR, western blot analysis and whole-embryo microRNA sensor assay in zebrafish. Recently, many studies have implicated miRNAs in various cardiovascular diseases. For example, miRNA-1-2 knockout mice have decreased levels of the cardiac transcription factor Hand2, which results in heart malformations (VSD) similar to those observed in Hand2-deficient mice $[53,54]$. Comparisons of malformed heart tissues from CHD patients with healthy controls have demonstrated dysregulation of miRNAs. However, to our knowledge, no evidence has shown that variations of miRNAs sequence, DNA binding motif or even altered miRNAs levels can directly cause CHD. Our study initially demonstrated that certain miRNAs can inhibit the expression of important transcription factors for cardiac development through the alternative binding sites and variable binding affinities, which eventually increase CHD risk. We also found that the levels of miR-30a and miR-9 were 3.72 and 4.91 times higher in CHD children compared with non-CHD adult controls. If it is true that miR-30a and miR-9 expression level changes are minimal after the heart has formed, our data suggest that miR-30a and miR-9 are likely contributors to the occurrence of CHDs. Because miRNAs are surprisingly stable and detectable in the circulating blood stream, they could serve as novel biomarkers for molecular diagnostics, as well as the promising therapeutic targets after they are validated by further studies. Therefore, future investigations on the interaction of CHD-associated regulatory variants in the master transcription factors and its target miRNAs will open a door for the improved CHD diagnosis and therapy.

In summary, our study demonstrated a significant effect of TBX5 3'UTR variant rs6489956 on the increased susceptibility of CHD in two independent Han Chinese populations. It was well known that both $T b \times 5$ overexpression and insufficiency result in heart defects in developing embryos. In view of the decreased $T B X 5 \mathrm{mRNA}$ levels detected in the heart tissues of CHD patients with the CT and TT genotypes, the individuals with the $\mathrm{T}$ allele are susceptible to develop CHD due to the TXB5 deficiency. Functional studies clearly showed that the consequent repression of $T B X 5$ expression on the post-transcriptional level resulted from an enhanced binding affinity of the $\mathrm{T}$ allele to miRNAs. Our data highlighted an important function of the underlying genetic variations in the $3^{\prime}$ UTR of $T B X 5$ in CHDs, providing new insight into risk assessment for these common birth defects. 
10

\section{Materials and Methods}

\section{Study subjects}

We analyzed samples from two independent case-control groups. The Shandong group consisted of $905 \mathrm{CHD}$ patients and 606 matched controls recruited from the Cardiovascular Disease Institute, General Hospital of Jinan Military Command (Jinan, Shandong Province, China) between August 2009 and September 2011. The Shanghai group was composed of 272 CHD cases and 384 matched controls enrolled in the Children's Hospital of Fudan University (Shanghai, China) between August 2010 and November 2011. All subjects were unrelated ethnic Han Chinese. All of the controls were non-CHD outpatients from the same geographical area who were matched to the affected individuals in terms of age and sex over the same period. CHDs patients who were considered to be syndromic and not isolated CHDs, or who had a positive family history of CHD in a first-degree relative (parents, siblings and children), were excluded from the study.

There were no newborn samples in our study. We classified the 1177 CHDs cases into seven broad categories according to the commonly accepted criteria [24]. Specifically, $156(13.3 \%)$ had conotruncal defects, $830(70.5 \%)$ had septal defects, 17 $(1.4 \%)$ had left ventricular outflow tract obstruction (LVOTO), $24(2.0 \%)$ had right ventricular outflow tract obstruction (RVOTO), 16 (1.4\%) had anomalous pulmonary venous return (APVR), 14 (1.2\%) had complex CHD and $120(10.2 \%)$ had other cardiac abnormalities (Supplementary Table S1).

To screen for genetic variants in the 3'UTR of TBX5 gene, we selected a subset of 32 subjects for resequencing. Half of these subjects were CHD patients, and half were controls. Fifty-three cardiovascular tissue samples were obtained with informed consent from CHD patients who had undergone cardiac operations from January 2011 to September 2011 in the Cardiovascular Disease Institute, General Hospital of Jinan Military Command (Jinan, Shandong Province, China) and Children's Hospital of Fudan University (Shanghai, China), among which 30 were used for quantitative real-time PCR (RT-PCR) assay and 23 for western blot analysis. The 53 collected cardiac tissue samples comprised 10 atrium samples, 4 ventricle samples, 4 appendage sample, 8 atrial septum samples, 4 ventricular septum samples, 15 outlet samples, and 8 aorta samples. Normal heart tissue samples were collected by the Department of Forensic Medicine, Fudan University from adults with no history of heart disease who died of accidents.

All study protocols were reviewed and approved by the medical ethics committee of Children's Hospital of Fudan University, and written consent was obtained from the parents of subjects and/or the subjects themselves before study enrollment.

\section{Variant identification and genotyping}

Genomic DNA was isolated from the peripheral venous blood using conventional regents. TBX5 3'UTR fragments were amplified using PCR to amplify a 1757-bp fragment from 114793401 to 114791645 (NM_000192) in 32 unrelated individuals randomly selected for variant screening by sequencing. Direct dye terminator sequencing of the PCR products was carried out using the ABI Prism BigDye system according to the manufacturer's instructions (Applied Biosystems, Foster City, CA, USA). Selected single-nucleotide polymorphisms (SNPs) with minor allele frequency (MAF) $>0.05$ were genotyped using $\mathrm{SNaPshot}$ analysis (ABI). In the first stage of the present study, a total of $288 \mathrm{CHD}$ cases and the same number of controls of the Shandong group were collected and genotyped in October 2010. The variants associated with CHD risk were chosen for further case-control study in the following Shandong and the Shanghai samples. The samples were sequenced and genotyped on an ABI 3730 automated sequencer and analyzed using Lasergene 7.0 and Peakscanner 1.0, respectively. All of the PCR and sequencing primers were listed in Supplementary Table S2.

\section{Quantitative real-time PCR}

Total RNA was extracted from human cardiovascular tissue samples preserved in RNAlater (Qiagen, Valencia, CA, USA) and converted to cDNA using random hexamers, oligo(dT) primers and PrimeScript RT reagent Kit (TaKaRa, Tokyo, Japan). Quantitative Real-time PCR was performed with SYBR Premix Ex Taq (TaKaRa) using a StepOne Real-Time PCR system (ABI) with $\beta$-actin as an internal reference gene. Each reaction was performed in triplicate. The primers were described in Supplementary Table S2.

\section{Western blot analysis}

Proteins were separated on $10 \%$ sodium dodecyl sulfate polyacrylamide electrophoresis gels and blotted on to polyvinylidene fluoride membranes (Millipore, Billerica, MA, USA). Non-specific binding was blocked with $5 \%$ skimmed milk in TBS. Blots were probed with either mouse horseradish peroxidase (HRP)-conjugated anti- $\beta$-actin monoclonal antibody (Kangchen Bio-tech, Shanghai, China), or rabbit anti-TBX5 polyclonal antibody (Abcam, Cambridge, UK) followed by HRP-conjugated goat anti-rabbit secondary antibody (Proteintech, Chicago, IL, USA). Proteins were detected using ECL reagents (Millipore).

\section{Plasmid}

To construct the reporter plasmids, TBX5 3'UTR containing either the $\mathrm{C}$ or $\mathrm{T}$ allele was amplified from genomic DNA and subcloned into the downstream region of Renilla luciferase using XhoI and NotI restriction sites in psiCHECK-2 vector (Promega, Madison, WI, USA). Primers were listed in Supplementary Table S2. All recombinant clones were verified by DNA sequencing.

Human wild-type TBX5 ORF fused to PCMV6-XL4 was purchased from Origene Technologies (Beijing, China). The mutant $T B X 5$ was generated by the the Quick Change SiteDirected Mutagenesis Kit (Stratagene). Constructs were linearized using $P v u \mathrm{II}$ and the capped and polyA tailed mRNA was synthesized with mMessage mMACHINE T7 Ultra Kit (Ambion, Life Technologies) for the zebrafish microinjection.

\section{Cell culture and luciferase reporter assays}

Human embryonic kidney 293T (HEK 293T), human cardiac myocytes $(\mathrm{HCM})$ and rat cardiac myocyte (H9C2) cells were 
grown in Dulbecco's Modified Eagle's Medium supplemented with $10 \%$ fetal bovine serum.

HEK 293T, HCM or H9C2 cells were seeded in 24-well culture plates at the density of $4.0 \times 10^{5}, 2.0 \times 10^{5}$ and $1.0 \times 10^{5}$ per liter, respectively. Twenty-four hours later, the cells were transfected with $400 \mathrm{ng}$ of each $T B X 5$ reporter plasmid using Lipofectamine 2000 (Invitrogen, Carlsbad, CA, USA). To screen the predicted candidate miRNAs, HEK 293T cells were co-transfected with $25 \mathrm{ng}$ of psiCHECK-C/T plasmid and $75 \mathrm{ng}$ of miRNA expression vector in 96-well culture plates. To further explore the interaction between TBX5 3'UTR and selected candidate miRNAs, HEK 293T, HCM or H9C2 cells were co-transfected with $50 \mathrm{ng}$ psiCHECK-C/T plasmid as well as $0 \mathrm{ng}, 50 \mathrm{ng}, 100 \mathrm{ng}, 150 \mathrm{ng}$ or $200 \mathrm{ng}$ of miRNA expression vector. For small interfering RNA assays, HEK 293T cells were co-transfected with $100 \mathrm{ng}$ psiCHECK-C/T plasmid and $100 \mathrm{~nm}$ miRNA inhibitors (RiboBio, Guangzhou, China) in 24-well culture plates.

Cells were lysed $24 \mathrm{~h}$ after transfection for luciferase assays that employed the Dual Luciferase Reporter Assay System (Promega) according to the manufacturer's instructions. Three independent transfection experiments were performed, and each luciferase assay was conducted in triplicate.

\section{Validation of $m i R N A$ regulation}

HEK $293 \mathrm{~T}$ cells were transfected with $2 \mu \mathrm{g}$ of miRNA expression vector or $100 \mathrm{~nm}$ of miRNA inhibitors in 6-well culture plates. Cells were harvested and lysed $48 \mathrm{~h}$ after transfection in RIPA buffer and assayed for the relative protein concentrations of intracellular TBX5 (rabbit anti-TBX5 polyclonal antibody; Abcam) and $\beta$-actin (Kangchen Bio-tech) using western blot analysis. RNA was also purified from these cells for quantitative RT-PCR analysis of TBX5 mRNA levels.

\section{Surface plasmon resonance}

SPR analysis was conducted using the ProteOn XPR36 protein interaction array system (Bio-Rad, Hercules, CA, USA). Biotinylated miRNAs were immobilized to an individual channel of the streptavidin-modified sensor chip at a fixed concentration of $400 \mathrm{~nm}$. Single-stranded RNA (GenePharma, Shanghai, China) harboring 32 bp of 3'UTR wild-type-C allele RNA or 3'UTR mutant-T-allele was diluted into a variety of concentrations in PBST buffer (10 mM Na $2 \mathrm{HPO}_{4}, 150 \mathrm{~mm} \mathrm{NaCl}$ and $0.005 \%$ Tween 20, pH 7.4) and flowed over the biotinylated miRNAs. At the end of each cycle, $5 \mathrm{~mm} \mathrm{NaOH}$ was applied to regenerate the sensor surface. Data were converted by BIA evaluation software.

\section{Zebrafish, microinjection and imaging}

The morpholino (MO) antisense oligonucleotide MO-Tbx5a (5'-GAAAGGTGTCTTCACTGTCCGCCAT-3') was purchased from Gene Tools (Philomatch, OR, USA). Human miR-30a, mir-9 and a negative control were purchased from Thermo Fisher Scientific (Waltham, MA, USA). Zebrafish were raised under standard conditions at $28.5^{\circ} \mathrm{C}$. Each 1-2-cell stage embryo was injected with a constant injection of $5 \mathrm{ng} \mathrm{MO}, 100 \mathrm{pg} T B X 5 \mathrm{mRNA}$ and $100 \mathrm{pg}$ miRNAs using a microinjector (Narishige, Japan). Twelve hours post-injection, the dead embryos were removed, leaving only viable embryos that were used for further analysis. Consistent with the previously published studies [18, 55], all live embryos were divided into the four categories according to their heart morphologies. At 48-h post fertilization (hpf), images were acquired with an Olympus stereomicroscope microscope or Leica TCS-SP5 LSM confocal microscope. For confocal imaging analysis of zebrafish embryos, they were anesthetized with egg water $/ 0.16 \mathrm{mg} \mathrm{ml}^{-1}$ tricaine/1\% 1- phenyl-2-thiourea (Sigma-Aldrich, St Louis, MO, USA) and embedded in $0.6 \%$ low melting agarose. Confocal imaging analysis was performed using Imaris software. Two transgenic zebrafish lines: $T g(v m h c: e G F P)$ and $T g$ (vmhc: $m$ Cherry-NTR) were used as described in previous work [55]. Whole-embryo microRNA sensor assay in zebrafish was carried out as described previously [56].

\section{Statistical analysis}

The Hardy-Weinberg equilibrium test was performed on controls using the $\chi^{2}$-test. Differences in demographic features and allelic or genotypic frequencies between the CHD cases and controls were evaluated using Pearson's $\chi^{2}$-test. To evaluate associations between the genotypes and CHD risk, ORs and 95\% CIs were calculated by unconditional logistic regression analysis with adjustments for age and sex. In multiple comparison testing, we employed the Bonferroni correction. The significance level was adjusted to $P=0.0025$ given the four SNPs being tested for 5 genetic models in 288 cases and the same number of controls.

Data were calculated as the mean \pm s.d. Significant differences between the test and control groups were analyzed by means of an independent $t$-test with Bonferroni correction. The $P<0.05$ was defined as statistical significance. Statistical analyses were conducted with SPSS 16.0 software (SPSS, Chicago, IL, USA).

\section{Conflict of Interest}

The authors declare no conflict of interest.

\section{Acknowledgements}

We sincerely thank all of the subjects who participated in this study and the clinicians who helped in recruiting the CHDs cases and controls. This work was supported by the 973 Program (2013CB945401), and the National Natural Science Foundation of China (81170147) to YHG; the National Natural Science Foundation of China (81430005, 81270232, 31521003), the 973 Program (2013CB945403), National key research and development program (2016YFC1000502), and the Commission for Science and Technology of Shanghai Municipality (13JC1407600) to HYW; the National Natural Science Foundation of China (81300126) to FW.

\section{Author contributions}

HYW and YHG conceived the project. FW, DL, RRZ, LWY and JYZ performed the experiments. BQ, FW, JYZ and $\mathrm{DM}$ contributed to the collection of clinical specimens and the 
analysis of human data. SSJ provided the microRNAs expression constructs. FW, DL, JYZ, XYY, FZ and LJ contributed to the design and interpretation of the experiments. FW and DL wrote the manuscript and all authors commented and approved the manuscript.

\section{References}

1 Bruneau BG. The developmental genetics of congenital heart disease. Nature 2008; 451: 943-948.

2 Basson CT, Bachinsky DR, Lin RC et al. Mutations in human TBX5 [corrected] cause limb and cardiac malformation in Holt-Oram syndrome. Nat Genet 1997; 15: 30-35.

3 Ching YH, Ghosh TK, Cross SJ et al. Mutation in myosin heavy chain 6 causes atrial septal defect. Nat Genet 2005; 37: 423-428.

4 Kirk EP, Sunde M, Costa MW et al. Mutations in cardiac T-box factor gene TBX20 are associated with diverse cardiac pathologies, including defects of septation and valvulogenesis and cardiomyopathy. Am J Hum Genet 2007; 81: 280-291.

5 Gutierrez-Roelens I, Sluysmans T, Gewillig M, Devriendt K, Vikkula M. Progressive AV-block and anomalous venous return among cardiac anomalies associated with two novel missense mutations in the CSX/NKX2-5 gene. Hum Mutation 2002; 20: 75-76.

6 Cordell HJ, Bentham J, Topf A et al. Genome-wide association study of multiple congenital heart disease phenotypes identifies a susceptibility locus for atrial septal defect at chromosome 4p16. Nat Genet 2013; 45: 822-824.

7 Kumar J, Yumnam S, Basu T et al. Association of polymorphisms in 9p21 region with CAD in North Indian population: replication of SNPs identified through GWAS. Clin Genet 2011; 79: 588-593.

8 Aouizerat BE, Vittinghoff E, Musone SL et al. GWAS for discovery and replication of genetic loci associated with sudden cardiac arrest in patients with coronary artery disease. BMC Cardiovasc Disord 2011; 11: 29.

9 Zhang L, Yin S, Miclaus K et al. Assessment of variability in GWAS with CRLMM genotyping algorithm on WTCCC coronary artery disease. Pharmacogenom J 2010; 10: $347-354$.

10 Rodriguez Esparragon F, Companioni O, Garcia Bello M, Buset Rios N, Rodriguez Perez JC. Replication of relevant SNPs associated with cardiovascular disease susceptibility obtained from GWAs in a case-control study in a Canarian population. Dis Markers 2012; 32: 231-239.

11 Zhao JY, Yang XY, Gong XH et al. Functional variant in methionine synthase reductase intron-1 significantly increases the risk of congenital heart disease in the Han Chinese population. Circulation 2012; 125: 482-490.

12 Zhao JY, Yang XY, Shi KH et al. A functional variant in the cystathionine beta-synthase gene promoter significantly reduces congenital heart disease susceptibility in a Han Chinese population. Cell Res 2013; 23: 242-253.
13 Zhao JY, Qiao B, Duan WY et al. Genetic variants reducing MTR gene expression increase the risk of congenital heart disease in Han Chinese populations. Eur Heart $J$ 2014; 35: 733-742.

14 Bruneau BG, Nemer G, Schmitt JP et al. A murine model of Holt-Oram syndrome defines roles of the T-box transcription factor Tbx 5 in cardiogenesis and disease. Cell 2001; 106: 709-721.

$15 \mathrm{Pu}$ WT, Ishiwata T, Juraszek AL, Ma Q, Izumo S. GATA4 is a dosage-sensitive regulator of cardiac morphogenesis. Dev Biol 2004; 275: 235-244.

16 Biben C, Weber R, Kesteven S et al. Cardiac septal and valvular dysmorphogenesis in mice heterozygous for mutations in the homeobox gene Nkx2-5. Circ Res 2000; 87: 888-895.

17 Li QY, Newbury-Ecob RA, Terrett JA et al. Holt-Oram syndrome is caused by mutations in TBX5, a member of the Brachyury (T) gene family. Nat Genet 1997; 15: 21-29.

18 Chiavacci E, Dolfi L, Verduci L et al. MicroRNA 218 mediates the effects of Tbx5a over-expression on zebrafish heart development. PLOS ONE 2012; 7: e50536.

19 Mori AD, Zhu Y, Vahora I et al. Tbx5-dependent rheostatic control of cardiac gene expression and morphogenesis. Dev Biol 2006; 297: 566-586.

20 Shan J, Pang S, Qiao Y et al. Functional analysis of the novel sequence variants within TBX5 gene promoter in patients with ventricular septal defects. Transl Res 2012; 160: 237-238.

21 Liu CX, Shen AD, Li XF et al. Association of TBX5 gene polymorphism with ventricular septal defect in the Chinese Han population. Chin Med J 2009; 122: 30-34.

22 Smemo S, Campos LC, Moskowitz IP, Krieger JE, Pereira AC, Nobrega MA. Regulatory variation in a TBX5 enhancer leads to isolated congenital heart disease. Hum Mol Genet 2012; 21: 3255-3263.

23 Wang F, Yang XY, Zhao JY et al. miR-10a and miR-10b target the 3'-untranslated region of TBX5 to repress its expression. Pediatr Cardiol 2014; 35 : 1072-1079.

24 Botto LD, Lin AE, Riehle-Colarusso T, Malik S, Correa A. Seeking causes: classifying and evaluating congenital heart defects in etiologic studies. Birth Defects Res Part A Clin Mol Teratol 2007; 79: 714-727.

25 Mishra PJ, Humeniuk R, Longo-Sorbello GS, Banerjee D, Bertino JR. A miR-24 microRNA binding-site polymorphism in dihydrofolate reductase gene leads to methotrexate resistance. Proc Natl Acad Sci USA 2007; 104: 13513-13518.

26 Garrity DM, Childs S, Fishman MC. The heartstrings mutation in zebrafish causes heart/fin Tbx 5 deficiency syndrome. Development 2002; 129: 4635-4645.

27 Rothschild SC, Easley CAT, Francescatto L, Lister JA, Garrity DM, Tombes RM. Tbx5-mediated expression of $\mathrm{Ca}(2+) /$ calmodulin-dependent protein kinase II is necessary for zebrafish cardiac and pectoral fin morphogenesis. Dev Biol 2009; 330: 175-184. 
28 Camarata T, Krcmery J, Snyder D, Park S, Topczewski J, Simon HG. Pdlim 7 (LMP4) regulation of Tbx 5 specifies zebrafish heart atrio-ventricular boundary and valve formation. Dev Biol 2010; 337: 233-245.

29 Pi-Roig A, Martin-Blanco E, Minguillon C. Distinct tissuespecific requirements for the zebrafish tbx 5 genes during heart, retina and pectoral fin development. Open Biol 2014; 4: 140014.

30 Li QY, NewburyEcob RA, Terrett JA et al. Holt-Oram syndrome is caused by mutations in TBX5, a member of the Brachyury (T) gene family. Nat Genet 1997; 15: 21-29.

31 Yang J, Hu D, Xia J et al. Three novel TBX5 mutations in Chinese patients with Holt-Oram syndrome. Am J Med Genet 2000; 92: 237-240.

32 Gruenauer-Kloevekorn C, Froster UG. Holt-Oram syndrome: a new mutation in the TBX5 gene in two unrelated families. Annal Genet 2003; 46: 19-23.

33 Fan C, Liu M, Wang Q. Functional analysis of TBX5 missense mutations associated with Holt-Oram syndrome. J Biol Chem 2003; 278: 8780-8785.

34 Ghosh TK, Packham EA, Bonser AJ, Robinson TE, Cross SJ, Brook JD. Characterization of the TBX5 binding site and analysis of mutations that cause Holt-Oram syndrome. Hum Mol Genet 2001; 10: 1983-1994.

35 Heinritz W, Moschik A, Kujat A et al. Identification of new mutations in the TBX5 gene in patients with Holt-Oram syndrome. Heart 2005; 91: 383-384.

36 Boogerd CJ, Dooijes D, Ilgun A et al. Functional analysis of novel TBX5 T-box mutations associated with HoltOram syndrome. Cardiovasc Res 2010; 88: 130-139.

37 Takeuchi JK, Ohgi M, Koshiba-Takeuchi K et al. Tbx5 specifies the left/right ventricles and ventricular septum position during cardiogenesis. Development 2003; 130: 5953-5964.

38 Brown DD, Martz SN, Binder O et al. Tbx5 and Tbx20 act synergistically to control vertebrate heart morphogenesis. Development 2005; 132: 553-563.

39 Zang X, Zhang S, Xia Y et al. SNP rs3825214 in TBX5 is associated with lone atrial fibrillation in Chinese Han population. PLOS ONE 2013; 8.

40 Smith JG, Magnani JW, Palmer C et al. Genome-wide association studies of the PR interval in African Americans. PLoS Genet 2011; 7: e1001304.

41 Hatcher CJ, Goldstein MM, Mah CS, Delia CS, Basson CT. Identification and localization of TBX5 transcription factor during human cardiac morphogenesis. Dev Dyn 2000; 219: 90-95.

42 Bruneau BG, Logan M, Davis N et al. Chamber-specific cardiac expression of Tbx 5 and heart defects in Holt-Oram syndrome. Dev Biol 1999; 211: 100-108.

43 Yamada M, Revelli JP, Eichele G, Barron M, Schwartz RJ. Expression of chick Tbx-2, Tbx-3, and Tbx-5 genes during early heart development: evidence for BMP2 induction of Tbx2. Dev Biol 2000; 228: 95-105.

44 Newbury-Ecob RA, Leanage R, Raeburn JA, Young ID. Holt-Oram syndrome: a clinical genetic study. $J$ Med Genet 1996; 33: 300-307.
45 Chaudhuri K, Chatterjee R. MicroRNA detection and target prediction: integration of computational and experimental approaches. DNA Cell Biol 2007; 26: 321-337.

46 van Rooij E, Sutherland LB, Qi X, Richardson JA, Hill J, Olson EN. Control of stress-dependent cardiac growth and gene expression by a microRNA. Science 2007; 316: 575-579.

47 Bernardo BC, Gao XM, Winbanks CE et al. Therapeutic inhibition of the miR-34 family attenuates pathological cardiac remodeling and improves heart function. Proc Natl Acad Sci USA 2012; 109: 17615-17620.

48 Divakaran V, Mann DL. The emerging role of microRNAs in cardiac remodeling and heart failure. Circ Res 2008; 103: 1072-1083.

49 Boettger T, Braun T. A new level of complexity: the role of microRNAs in cardiovascular development. Circ Res 2012; 110: 1000-1013.

50 Li D, Ji L, Liu L et al. Characterization of circulating microRNA expression in patients with a ventricular septal defect. PLoS ONE 2014; 9: e106318.

51 Liang D, Xu X, Deng F et al. miRNA-940 reduction contributes to human Tetralogy of Fallot development. J Cell Mol Med 2014; 18: 1830-1839.

52 Bittel DC, Kibiryeva N, Marshall JA, O'Brien JE. MicroRNA-421 dysregulation is associated with tetralogy of Fallot. Cells 2014; 3: 713-723.

53 Zhao Y, Samal E, Srivastava D. Serum response factor regulates a muscle-specific microRNA that targets Hand2 during cardiogenesis. Nature 2005; 436: 214-220.

54 Zhao Y, Ransom JF, Li A et al. Dysregulation of cardiogenesis, cardiac conduction, and cell cycle in mice lacking miRNA-1-2. Cell 2007; 129: 303-317.

55 Zhang $\mathrm{R}$, Han $\mathrm{P}$, Yang $\mathrm{H}$ et al. In vivo cardiac reprogramming contributes to zebrafish heart regeneration. Nature 2013; 498: 497-501.

56 Wang X, Ling CC, Li L et al. MicroRNA-10a/10b represses a novel target gene mib1 to regulate angiogenesis. Cardiovasc Res 2016; 110: 140-150.

(Supplementary information is linked to the online version of the paper on the Cell Discovery website.)

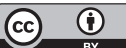

This work is licensed under a Creative Commons Attribution 4.0 International License. The images or other third party material in this article are included in the article's Creative Commons license, unless indicated otherwise in the credit line; if the material is not included under the Creative Commons license, users will need to obtain permission from the license holder to reproduce the material. To view a copy of this license, visit http://creativecommons.org/licenses/by/4.0/

(C) The Author(s) 2017 